\title{
TM-25659-Induced Activation of FGF21 Level Decreases Insulin Resistance and Inflammation in Skeletal Muscle via GCN2 Pathways
}

\author{
Jong Gab Jung ${ }^{1}$, Sang-A Yi ${ }^{1}$, Sung-E Choí, Yup Kang', Tae Ho Kim ${ }^{3}$, Ja Young Jeon', Myung Ae Bae ${ }^{4}$, Jin \\ Hee Ahn $n^{4}$, Hana Jeong ${ }^{5}$, Eun Sook Hwang ${ }^{5}$, and Kwan-Woo Lee ${ }^{1, *}$
}

The TAZ activator 2-butyl-5-methyl-6-(pyridine-3-yl)-3[2'-(1H-tetrazole-5-yl)-biphenyl-4-yImethyl]-3H-imidazo[4,5b]pyridine] (TM-25659) inhibits adipocyte differentiation by interacting with peroxisome proliferator-activated receptor gamma. TM-25659 was previously shown to decrease weight gain in a high fat (HF) diet-induced obesity (DIO) mouse model. However, the fundamental mechanisms underlying the effects of TM-25659 remain unknown. Therefore, we investigated the effects of TM-25659 on skeletal muscle functions in $\mathrm{C2}$ myotubes and C57BL/6J mice. We studied the molecular mechanisms underlying the contribution of TM-25659 to palmitate (PA)-induced insulin resistance in C2 myotubes. TM-25659 improved PAinduced insulin resistance and inflammation in C2 myotubes. In addition, TM-25659 increased FGF21 mRNA expression, protein levels, and FGF21 secretion in C2 myotubes via activation of GCN2 pathways (GCN2-phosphoeIF2 $\alpha$-ATF4 and FGF21). This beneficial effect of TM-25659 was diminished by FGF21 siRNA. C57BL/6J mice were fed a HF diet for 30 weeks. The HF-diet group was randomly divided into two groups for the next 14 days: the HF-diet and HF-diet + TM-25659 groups. The HF diet + TM-25659treated mice showed improvements in their fasting blood glucose levels, insulin sensitivity, insulin-stimulated Akt phosphorylation, and inflammation, but neither body weight nor food intake was affected. The HF diet + TM25659-treated mice also exhibited increased expression of both FGF21 mRNA and protein. These data indicate that

${ }^{1}$ Department of Endocrinology and Metabolism, ${ }^{2}$ Department of Physiology, Ajou University School of Medicine, Suwon 443-749, Korea, ${ }^{3}$ Division of Endocrine and Metabolism, Department of Internal Medicine, Seoul Medical Center, Seoul 138-160, Korea, ${ }^{4}$ Korea Research Institute of Chemical Technology, University of Science \& Technology, Daejeon 305-600, Korea, ${ }^{5}$ College of Pharmacy, Graduate School of Pharmaceutical Sciences, and Global Top5 Research Program, Ewha Womans University, Seoul 120-750, Korea

*Correspondence: Ikw65@ajou.ac.kr

Received 9 April, 2015; revised 24 August, 2015; accepted 8 September, 2015; published online 4 November, 2015

Keywords: FGF21, GCN2, inflammation, insulin resistance, TM-25659
TM-25659 may be beneficial for treating insulin resistance by inducing FGF21 in models of PA-induced insulin resistance and HF diet-induced insulin resistance.

\section{INTRODUCTION}

Transcriptional co-activator with PDZ-binding motif (TAZ) functions as a co-activator, and its activity is regulated via interactions with 14-3-3 protein and PDZ domain proteins (Kanai et al. 2000). Increased TAZ activity in the nucleus suppresses adipocyte development and increases osteoblast differentiation by interacting with peroxisome proliferator-activated receptor gamma (PPAR- $\gamma$ ) and runt-related transcription factor 2, respectively (Hong et al., 2005). The TAZ activator 2-butyl-5methyl-6-(pyridine-3-yl)-3-[2'-(1H-tetrazole-5-yl)-biphenyl-4ylmethyl]-3H-imidazo[4,5-b]pyridine] (TM-25659) inhibits adipocyte development by interacting with PPAR- $\gamma$ and decreases weight gain in obese mouse models (Jang et al., 2012). However, the fundamental mechanisms underlying the effects of TM-25659 are unknown.

Fibroblast growth factor 21 (FGF21) is a member of the FGF family with multiple metabolic functions (Kharitonenkov et al., 2005). FGF21 functions as an endocrine hormone with antidiabetic and antiobesity effects and is produced in peripheral tissues (e.g., the liver, white and brown adipose tissues, skeletal muscle, and the pancreas) (Fisher et al., 2010). Specifically, the administration of recombinant FGF21 or overexpression of FGF21 ameliorates obesity, insulin sensitivity, inflammation, and glucose clearance in obese or diabetic animals (Kharitonenkov et al., 2005; Wang et al., 2015; Xu et al., 2009). In contrast, FGF21 deficiency leads to body weight gain, impaired glucose tolerance, elevated blood insulin, and fatty liver development (Badman et al., 2007; Inagaki et al., 2007).

General control nonderepressible 2 (GCN2) is a serine/ threonine protein kinase used to detect amino acid deficiency. Decreased consumption of dietary protein leads to a reduction in the amount of amino acids delivered to the liver, activation of GCN2, and an eventual increase in elF2- $\alpha$ phosphorylation and activation of ATF4 (Anthony et al., 2004; Laeger et al., 2014). ATF4 binds amino acid response elements (AAREs) in the FGF21 promoter, which leads to an increase of FGF21 in the liver and blood circulation. FGF21 increases energy 
expenditure (EE) and decreases body weight gain in mice (Ge et al., 2012; Laeger et al., 2014). However, GCN2-knockout mice fail to exhibit increased elF2 $\alpha$ phosphorylation during both dietary and pharmacological amino acid deprivation, ultimately resulting in hepatic steatosis and liver injury (Anthony et al., 2004; Guo and Cavener, 2007; Wilson et al.,2013).

We found that TM-25659 improved insulin signaling and inflammation in PA-induced insulin-resistant skeletal muscle cells. TM-25659 also increased FGF21 mRNA, protein, and secretion levels in association with increased GCN2 in C2 myotubes. The TM-25659-increased FGF21 protein levels were reduced by GCN2 siRNA. Additionally, administration of TM-25659 lowered the fasting glucose levels, improved insulin resistance, ameliorated inflammation, and increased FGF21 protein levels in our HF diet-induced obesity (DIO) mouse model.

In this study, we evaluated the reciprocal relationship between TM-25659 and FGF21 in skeletal muscle cells and mice, and examined the hypothesis that FGF21 mediates the metabolic benefits of a TM-25659 by comparing the effects of TM25659-treated skeletal muscle cells subjected to the small interfering RNA (siRNA)-mediated knockdown of FGF21 (FGF21 siRNA) using PA-induced insulin-resistant skeletal muscle cells as controls.

\section{MATERIALS AND METHODS}

\section{Materials}

TM-25659 was obtained from the Korea Research Institute of Chemical Technology (Korea). Bovine serum albumin (BSA), insulin, PA, and methyl cellulose were purchased from SigmaAldrich (USA). Anti-AKT, anti-phospho-AKT (ser473), antielF2 $\alpha$, anti-phospho-elF2 $\alpha$ (ser51), anti-PERK, anti-phosphoPERK (Thr980), and anti-ATF4 antibodies were acquired from Cell Signaling Technology (USA). Anti-GCN2, anti-phosphoGCN2 (Thr898) antibodies were acquired from Biorbyt (UK). Anti-FGF21 antibodies were purchased from Abcam (USA). Anti-actin antibody was obtained from Santa Cruz Biotechnology (USA). Culture media, culture supplements, horse serum, and fetal bovine serum (FBS) were purchased from Gibco-BRL (USA). TM-25659 was dissolved in $0.5 \%$ methyl cellulose in water for in vivo administration.

\section{Preparation of PA}

PA/BSA conjugates were prepared by soaping PA with sodium hydroxide $(\mathrm{NaOH})$ and mixing with BSA. Briefly, $20 \mathrm{mM}$ PA in $10 \mathrm{mM} \mathrm{NaOH}$ was incubated at $70^{\circ} \mathrm{C}$ for $30 \mathrm{~min}$, and the fatty acid soaps were combined with $5 \%$ fatty acid-free BSA in phosphate-buffered saline (PBS) at a 1:3 volume ratio. The combined fatty acids consisted of $5 \mathrm{mM}$ PA and $3.75 \%$ BSA. The PA/BSA conjugates were diluted in $5 \%$ horse serum medium ( $0.4 \%$ BSA) and administered to cultured cells at a final concentration of $500 \mu \mathrm{M}$ PA. The molar ratio of PA to BSA was $\sim 3.5: 1$, and the concentration of BSA in the $500 \mu \mathrm{M}$ PA medium was $\sim 0.7 \%$.

\section{Cell culture}

C2 mouse skeletal myoblasts were obtained from Dr. Hey-Sun Kim (Ajou University, Korea) and grown in high-glucose (4.5 g/L glucose) Dulbecco's modified Eagle's medium (DMEM) supplemented with $10 \%$ FBS and antibiotics $(10 \mu \mathrm{g} / \mathrm{ml}$ streptomycin and $100 \mathrm{IU} / \mathrm{ml}$ penicillin) at $37^{\circ} \mathrm{C}$ in a humidified atmosphere of $95 \%$ air and $5 \% \mathrm{CO}_{2}$. After washing the $70-80 \%$ confluent myoblasts with PBS, differentiation to myotubes was initiated by transferring the cells to low-serum differentiation medium [DMEM with low glucose $(1 \mathrm{~g} / \mathrm{L})$ supplemented with $5 \%$ horse serum]. After a 3-day incubation, differentiation was complete as determined by morphological changes and creatine kinase expression.

\section{Immunoblot analysis}

Cells and mouse soleus muscles were suspended in RIPA buffer [150 mM sodium chloride ( $\mathrm{NaCl}), 1 \% \mathrm{NP}-40,0.5 \%$ deoxycholate, $0.1 \%$ sodium dodecyl sulfate (SDS), $50 \mathrm{mM}$ Tris-HCl, $\mathrm{pH} 7.5$, and protease inhibitor cocktail (Roche Applied Science, Germany)] and incubated on ice for $20 \mathrm{~min}$. Whole proteins were extracted by differential centrifugation $(13,000 \times g, 10 \mathrm{~min})$ and the protein concentrations in the lysates were determined using a protein assay kit (Bio-Rad, USA). An equal volume of $2 \times$ SDS sample buffer (125 mM Tris-HCl, pH 6.8, 4\% SDS, 4\% 2-mercaptoethanol, and $20 \%$ glycerol) was added to the cell lysates, and equivalent amounts of protein $(20 \mu \mathrm{g})$ were loaded onto $8-12 \%$ polyacrylamide gels, electrophoresed, and transferred electrophoretically to polyvinylidene fluoride membranes (Millipore, USA). After blocking the membranes with 5\% skim milk for $30 \mathrm{~min}$, the target antigens were reacted with primary antibodies, followed by secondary antibodies (horseradish peroxidase-conjugated anti-goat IgG or anti-rabbit lgG). Immunoreactive bands were visualized by enhanced chemiluminescence (Amersham Pharmacia Biotech, USA).

RNA isolation and quantitative real-time polymerase chain reaction (PCR)

Total RNA was isolated from cell or muscle tissues and prepared using RNAiso Plus reagent (TaKaRa Bio Inc., Japan) according to the manufacturer's instructions. Briefly, C2 myotube cDNA was synthesized with avian myeloblastosis virus reverse transcriptase using random 9-mers. The cDNA was amplified by PCR with primer sets for FGF21 [CCC CAA ACC AGG ATG CAA CA (forward, F) and ATG CTG CTT GGT CTT GGG GC (reverse, R)], tumor necrosis factor (TNF)- $\alpha$ [GGC ACT CCC CCA AAA GAT GG (F) and CCC TGC CAC AAG CAG GAA TG (R)], interleukin (IL)-6 [CCA TCC AGT TGC CTT CTT GGG (F) and GCC GTG GTT GTC ACC AGC AT (R)], IL$1 \beta$ [TCT CGC AGC AGC ACA TCA ACA (F) and CCT GGA AGG TCC ACG GGA AA (R)], monocyte chemoattractant protein (MCP)-1 [CAG CCA GAT GCA GTT AAC GC (F) and GCC TAC TCA TTG GGA TCA TCT (R)], and RPL32 [AAG CGA AAC TGG CGG AAA CC (F) and CCC ATA ACC GAT GTT GGG CA (R)]. Quantitative real-time PCR was performed using SYBR Green (TaKaRa Bio Inc.) with a TaKaRa TP-815 instrument. All expression values were normalized to RPL32 mRNA levels.

\section{siRNAs}

Twenty-one nucleotide siRNA duplexes were designed and created by Bioneer (Korea). The siRNA sequences were as follows: green fluorescent protein, 5'-GUU CAG CGU GUC CGG CGA GTT-3', FGF21, 5'-CUG AUG GAA UGG AUG AGA U-3', and GCN2, 5'-GUG GAA GCU AAA UUC UGU A-3'. C2 myotubes were transfected with siRNA oligonucleotides using a pipette-type electroporator (Microporator-Mini; Digital Biotechnology, Korea) according to the manufacturer's instructions. Briefly, $1 \times 10^{6} \mathrm{C} 2$ myotubes were transfected with $60 \mathrm{nM}$ siRNA ( $1 \mu \mathrm{g}$ in $10 \mu \mathrm{l}$ of $\mathrm{R}$ buffer) by microporation at a pulse voltage of $1005 \mathrm{~V}$, pulse width of $35 \mathrm{~ms}$, and pulse number of 2 . After transfection, the C2 myotubes were differentiated by switching the medium to differentiation medium for 3 days. Following differentiation, the cells were seeded in 12-well plates at a concentration of $1 \times 10^{5}$ cells/well and treated with or without TM-25659 for $16 \mathrm{~h}$. 
Uptake of 2-N-(7-nitrobenz-2-oxa-1,3-diazol-4-yl) amino-2deoxyglucose (2-NBDG)

C2 myotubes were pretreated with PA $(500 \mu \mathrm{M})$ and TM$25659(25$ or $50 \mu \mathrm{M})$ for $16 \mathrm{~h}$. Next, the cells were starved for $4 \mathrm{~h}$ and then preincubated in Krebs-Ringer bicarbonate buffer (pH 7.4) containing $2 \% \mathrm{BSA}$ at $37^{\circ} \mathrm{C}$ for $30 \mathrm{~min}$. They were then treated with $500 \mu \mathrm{M} 2-\mathrm{NBDG}$ (cat. no. N13195; Invitrogen, USA) with or without $100 \mathrm{nM}$ insulin at $37^{\circ} \mathrm{C}$ for $2 \mathrm{~h}$. The cells were washed with ice-cold PBS three times and lysed with cell lysis buffer. The lysates were centrifuged at 13,000 $\mathrm{rpm}$ and $4^{\circ} \mathrm{C}$ for $20 \mathrm{~min}$. The supernatants were measured for fluorescence (excitation $475 \mathrm{~nm}$, emission $550 \mathrm{~nm}$ ) using a SpectraMax Gemini EM microplate reader (Molecular Devices, USA). Protein concentrations were determined by the Bradford assay.

\section{Animal experiments}

Six-week-old male C57BL/6J mice were purchased from Japan SLC Inc. (Japan). All animal experiments were approved by the Animal Ethics Committee of the Laboratory Animal Research Center, Ajou University Medical Center (Korea). The mice were housed in a temperature-controlled room (22 $\pm 2^{\circ} \mathrm{C}$ ) with a 12/12-h light/dark cycle and fed ad libitum. Eight-week-old male C57BL/6J mice were fed a HF diet $(n=$ 12) for 30 weeks. The mice were provided a HF diet with $60 \%$ kcal from fat (Research Diets, USA) ad libitum. The HF diet group was randomly assigned during the additional 14 days to two groups: HF and HF + $50 \mathrm{mg} / \mathrm{kg}$ TM-25659 (TM). The mice were orally administered either vehicle or TM-25659 (50 $\mathrm{mg} / \mathrm{kg}$ ) every other day for 14 days, and their body weight was measured every other day.

\section{Insulin tolerance tests}

The intraperitoneal insulin tolerance test (ITT) was performed by intraperitoneally injecting regular human insulin $(0.5 \mathrm{U} / \mathrm{kg})$ after a 6-h fast. Blood samples were taken at different time points $(0,30,60$, and $120 \mathrm{~min}$ after insulin loading) from a tail vein. Plasma glucose was measured using Accu-chek (Korea Roche Diagnostics, Korea).

\section{FGF21 secretion}

The concentration of FGF21 in the culture medium of C2 myotubes was determined using a mouse/rat FGF21 enzymelinked immunosorbent assay (ELISA) (R\&D Systems, USA). The concentration of albumin in the culture medium was determined using a mouse albumin ELISA kit (BioTek, USA).

\section{Statistical analysis}

Data are presented as the mean \pm standard error of at least three independent experiments. Statistical differences between the groups were determined using Student's $t$-test and Fisher's exact test. $P$-values $<0.05$ were considered significant.
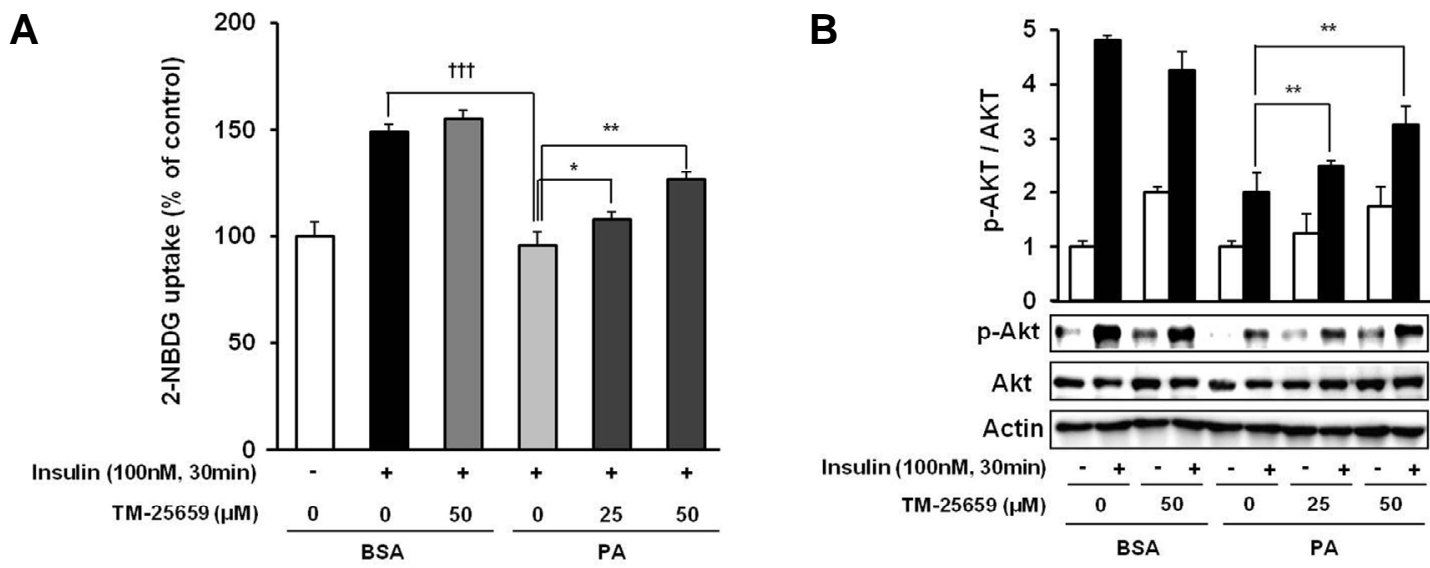

C

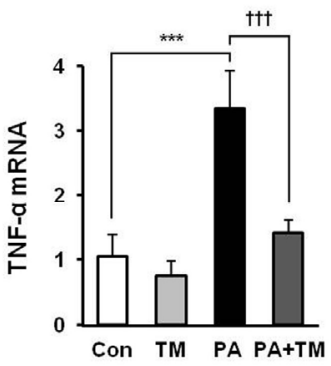

$\boldsymbol{D}$

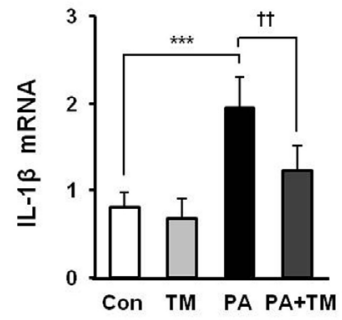

$E$

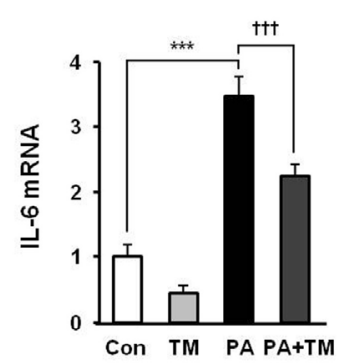

$\boldsymbol{F}$

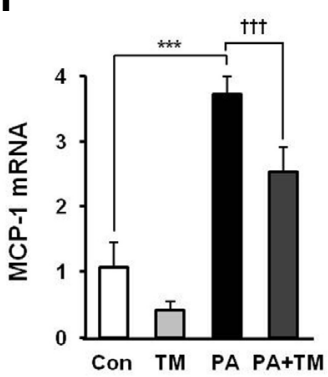

Fig. 1. Beneficial effects of TM-25659 on insulin resistance and inflammation caused by palmitate (PA) in skeletal muscle cells. Skeletal muscle cells were harvested 30 min after insulin (100 nM) treatment of PA-, TM-25659-, or vehicle-treated cells for $16 \mathrm{~h}$. (A) TM-25659 prevented PA-induced impaired glucose uptake by 2-NBDG in C2 myotubes. (B) Representative immunoblots showing phospho (Ser 473) and total AKT in skeletal muscle cells of the control (Con), TAZ modulator (TM), PA, and PA + TM groups, respectively. (C-F) TNF- $\alpha$, IL-1 $\beta$, IL-6, and MCP-1 mRNA expression as quantified by real-time PCR. [Con, BSA; TM, $50 \mu \mathrm{M} \mathrm{TM-25659;} \mathrm{PA,} 500 \mu \mathrm{M}$ PA for $16 \mathrm{~h}$ ]. The data are given as the mean \pm standard error. ${ }^{\star} P<0.05,{ }^{\star \star} P<0.01,{ }^{* \star} P<0.001, \dagger^{\dagger} P P<0.01, \dagger_{\dagger} \dagger P<0.001$ 
A

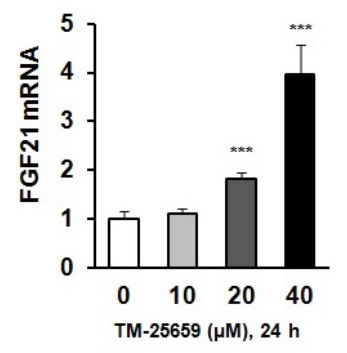

D

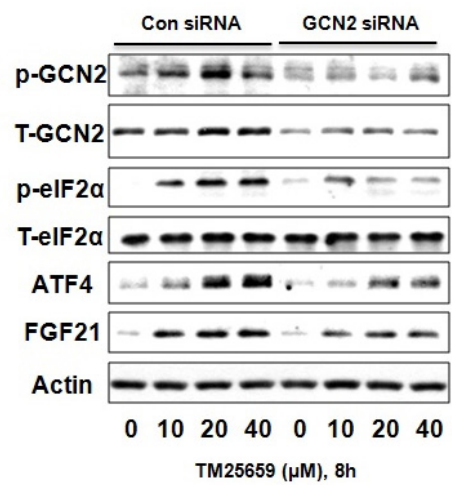

\section{RESULTS}

\section{TM-25659 restored PA-induced insulin resistance and} inflammation in skeletal muscle cells

In previous experiments, we found $500 \mu \mathrm{M}$ PA decreased insulin-stimulated glucose uptake in C2 myotubes (Jung et al., 2011). In this study, we investigated the effects of TM-25659 on PA-induced insulin resistance in C2 myotubes. TM-25659 prevented the decrease in insulin-stimulated glucose uptake in C2 myotubes (Fig. 1A). Additionally, we investigated the effects of TM-25659 on the reduction of insulin-stimulated Akt phosphorylation by PA in C2 myotubes. TM-25659 significantly inhibited the PA-induced reduction in insulin-stimulated Akt phosphorylation (Fig. 1B). These data suggest that TM-25659 restored the PA-induced decrease in insulin sensitivity.

PA increased pro-inflammatory cytokine gene expression in C2 myotubes, while TM-25659 markedly decreased PAinduced expression of TNF- $\alpha, \mathrm{IL}-1 \beta, \mathrm{IL}-6$, and MCP-1 in $\mathrm{C} 2$ myotubes (Figs. 1C-1F). Additionally, the PA-induced IL-1 $\beta$ maturation levels decreased in C2 myotubes in the PA + TM25659 (TM) group (data not shown). These data demonstrate that TM-25659 prevented the PA-induced expression of proinflammatory cytokines in $\mathrm{C} 2$ myotubes.

\section{TM-25659 increased FGF21 levels by GCN2 pathways in skeletal muscle cells}

We found that TM-25659 increased FGF21 mRNA, protein, and secretion levels in C2 myotubes. Importantly, TM-25659 significantly increased FGF21 mRNA expression in C2 myotubes in a dose-dependent manner (Fig. 2A). We also observed increased FGF21 protein levels in C2 myotubes treated with TM-25659 (Fig. 2B). Moreover, TM-25659 increased FGF21 secretion in C2 myotubes (Fig. 2C).

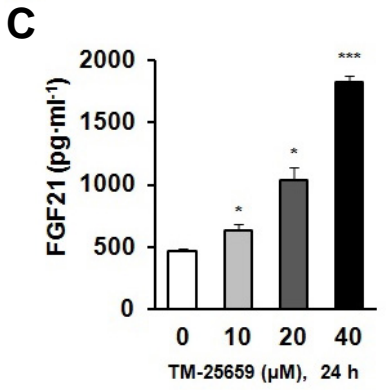

Fig. 2. Effects of TM-25659 on FGF21 expression in skeletal muscle cells. C2 myoblasts were differentiated into $\mathrm{C} 2$ myotubes and treated with 10,20 , or $40 \mu \mathrm{M}$ of TM-25659 or vehicle. (A) FGF21 mRNA expression levels as quantified by real-time PCR. (B) FGF21 protein levels as determined by immunoblotting. (C) Secretion of FGF21 as quantified by an enzyme-linked immunosorbent assay (ELISA). (D) GCN pathway in TM-25659-treated skeletal muscle cells with or without GCN2 siRNA. The data are given as the mean \pm standard error. ${ }^{*} P<0.05,{ }^{* \star} P<0.01,{ }^{\star \star \star} P<$ $0.001, \uparrow \dagger P<0.01, \uparrow \dagger \dagger P<0.001$.
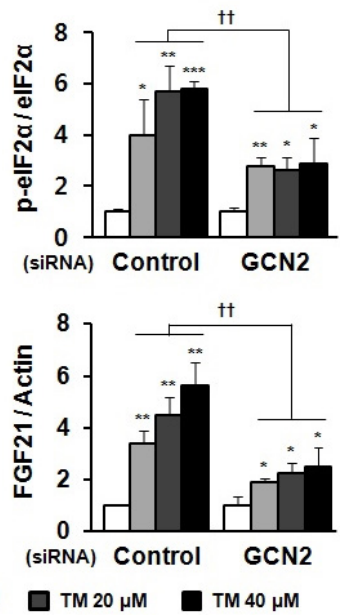

TM-25659-induced increased FGF21 protein levels through GCN2 pathways (GCN2-phospho-elF2 $\alpha$-ATF4 and FGF21) (Fig. 2D). Therefore, we next investigated whether TM-25659 increased GCN2 pathways in C2 myotubes that were and were not treated with GCN2 siRNA. We found that TM-25659 induced GCN2 pathways, but this induction was diminished by GCN2 siRNA (Fig. 2D). We also investigated the involvement of other pathways i.r., PERK and PKR, in TM-25659-induced FGF21 expression. We found that TM-25659 induced the induction of PERK and PKR, while FGF21 induction was not reduced by PERK or PKR siRNA treatment. (Supplementary Fig. 1). These data indicate TM-25659-induced increases in FGF21 levels through GCN2 pathways in C2 myotubes.

Effects of TM-25659-reduced insulin resistance and inflammation in skeletal muscle cells treated with or without FGF21 SIRNA

We previously showed that TM-25659 markedly increased FGF21 mRNA and protein expression in TM-25659-treated skeletal muscle cells (Figs. 2A and $2 \mathrm{~B}$ ). We investigated the effects of TM-25659-reduced insulin resistance and inflammation in C2 myotubes with or without FGF21 siRNA treatment. FGF21 mRNA and protein expression decreased following FGF21 siRNA treatment (Figs. 3A and 3B). TM-25659 prevented the PA-induced decrease in insulin-stimulated Akt phosphorylation, and the beneficial effects of TM-25659 decreased following FGF21 siRNA treatment (Fig. 3C). We next investigated whether TM-25659 prevented the PA-increased levels of pro-inflammatory cytokines in C2 myotubes treated with or without FGF21 siRNA. We found that the beneficial effects of TM-25659 prevented PA-increased pro-inflammatory cytokine expression (MCP-1, TNF- $\alpha$, IL-1 $\beta$, and IL-6), but the decrease in induction was lowered by FGF21 siRNA (Fig. 3D). Consistent 
$\boldsymbol{A}$

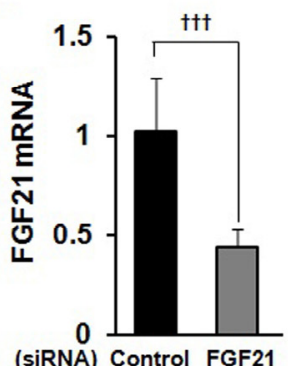

(siRNA) Control FGF21
B

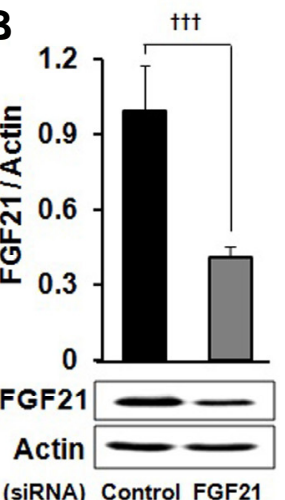

$C$

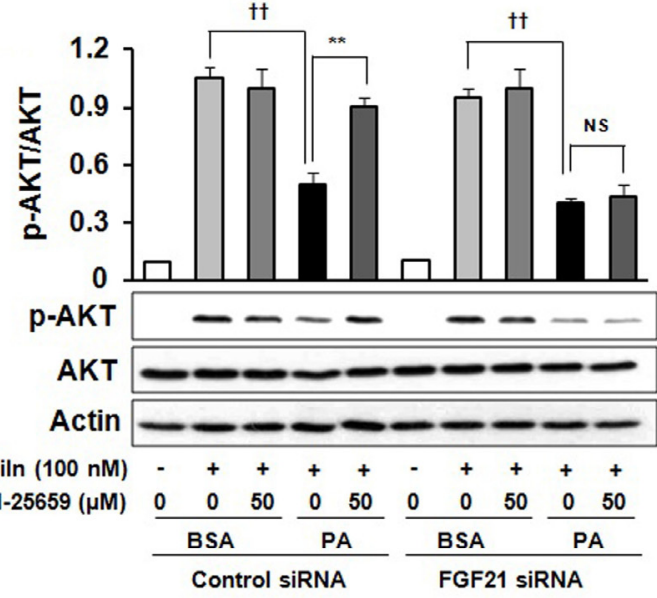

$\boldsymbol{D}$
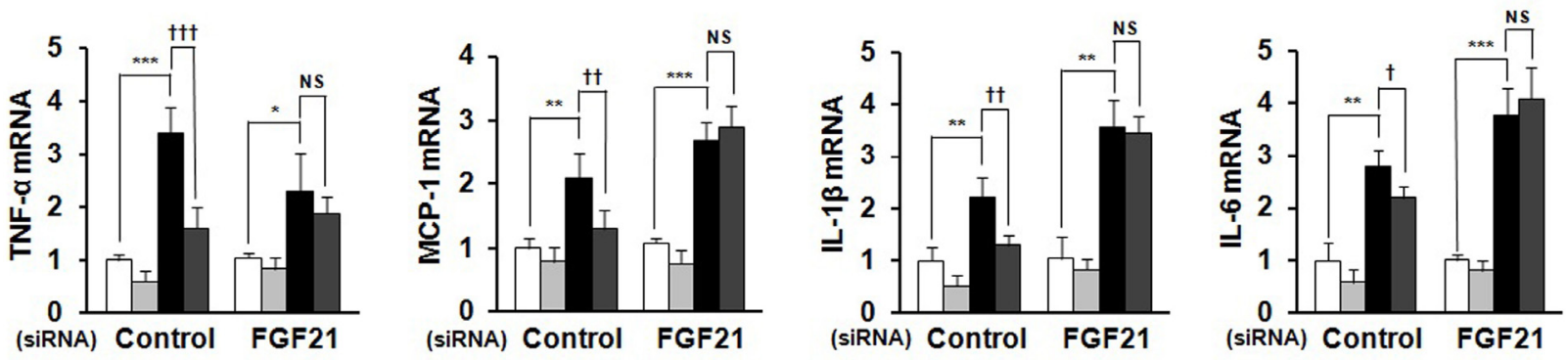

$\square$ Con

TM

PA

$\mathrm{PA}+\mathrm{TM}$

Fig. 3. TM-25659 inhibited insulin resistance and inflammation in skeletal muscle cells in an FGF21-dependent manner. (A) FGF21 mRNA expression with or without FGF21 siRNA as quantified by real-time PCR. (B) Representative immunoblots showing FGF21 in skeletal muscle cells with or without FGF21 siRNA. (C) Representative immunoblots showing phospho (Ser 473) and total AKT in skeletal muscle cells of the control (BSA) and palmitate (PA) groups with or without FGF21. (D) TNF- $\alpha$, IL-1 $\beta$, IL-6, and MCP-1 mRNA expression with or without FGF21 siRNA as quantified by real-time PCR (control (Con), TM-25659 (TM), palmitate (PA), and PA + TM). The data are given as the mean \pm standard error. ${ }^{*} P<0.05,{ }^{* \star} P<0.01,{ }^{* \star *} P<0.001, \dagger P<0.05, \dagger \dagger P<0.01, \dagger_{\dagger}+P<0.001$.

with our observations in C2 myotubes, TM-25659 significantly decreased the PA-induced expression of pro-inflammatory cytokines in C2 myotubes, whereas these effects were abolished by FGF21 siRNA.

\section{TM-25659 regulates insulin sensitivity and FGF21 mRNA and protein expression in DIO mice}

The mice were fed a HF diet for 30 weeks to examine the metabolic effects of TM-25659 treatment before any effects occurred on body weight. Mice in the HF-diet group were randomly divided into two groups for the next 14 days: the HF-diet and HF-diet + TM-25659 groups. After 14 days, no significant changes were observed in body weight or food intake (Figs. 4A and $4 \mathrm{~B}$ ).

The glucose level in the HF + TM group decreased from 9.8 \pm 0.5 to $7.6 \pm 0.4 \mathrm{mg} / \mathrm{dl}$ with fasting (Fig. 4C). TM-25659 treatment improved insulin sensitivity after 14 days, as evaluated by a fasting blood glucose and ITT; a significant decrease in the glucose level occurred postinjection (Fig. 4D).

TM-25659 prevented the HF diet-induced decrease in insulin-stimulated Akt phosphorylation in mouse skeletal muscles (Fig. 4E). We next investigated whether TM-25659 prevented the HF diet-induced increases in the pro-inflammatory cytokine levels in mouse skeletal muscles. We found beneficial effects of
TM-25659 in that it decreased the HF diet-increased proinflammatory cytokine expression levels (MCP-1, TNF- $\alpha$, and IL-1) (Fig. 4F). Because FGF21 was markedly expressed in skeletal muscle cells (C2 myotubes), we investigated the effect of TM-25659 on FGF21 expression in mouse skeletal muscle. After TM-25659 treatment, the FGF21 mRNA expression significantly increased by $>2.5$-fold, and the FGF21 protein level significantly increased by $>1$.8-fold (Figs. $4 \mathrm{G}$ and $4 \mathrm{H}$ ). Therefore, TM-25659 is able to induce FGF21 expression and insulin sensitivity without changes in body weight.

\section{DISCUSSION}

In this study, treating skeletal insulin-resistant muscle cells with the TAZ activator TM-25659 protected against insulin resistance and inflammation. TM-25659 increased the FGF21 mRNA and protein levels in HF diet-induced mouse skeletal muscle and C2 myotubes. Additionally, TM-25659 increased FGF21 mRNA expression and stimulated FGF21 secretion in C2 myotubes. When skeletal muscle cells were treated with FGF21 siRNA, TM-25659 did not protect against PA-induced insulin resistance and inflammation. Additionally, we demonstrated that TM-25659 administration to HF-induced DIO mice improved the fasting glucose level and insulin sensitivity. Thus, 
$\boldsymbol{A}$

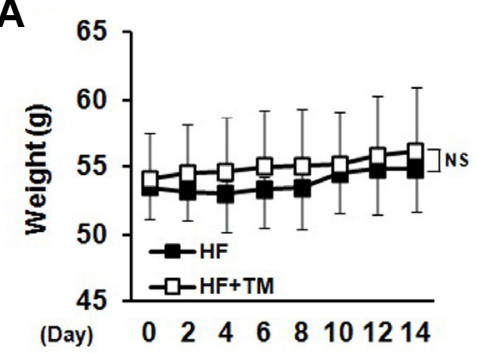

$B$

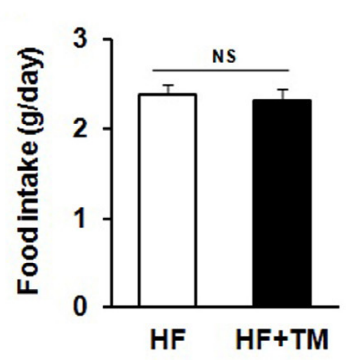

C

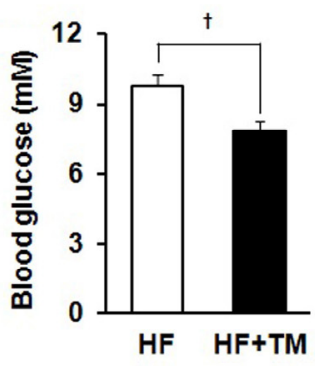

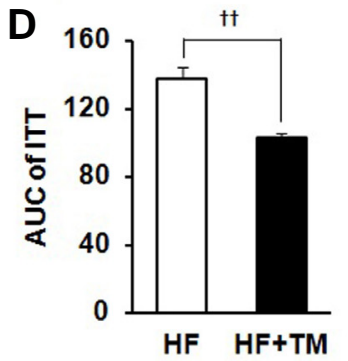

$\boldsymbol{H}$
E

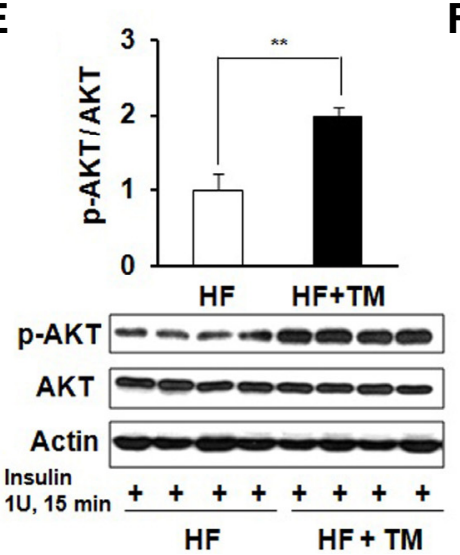

$\boldsymbol{F}$

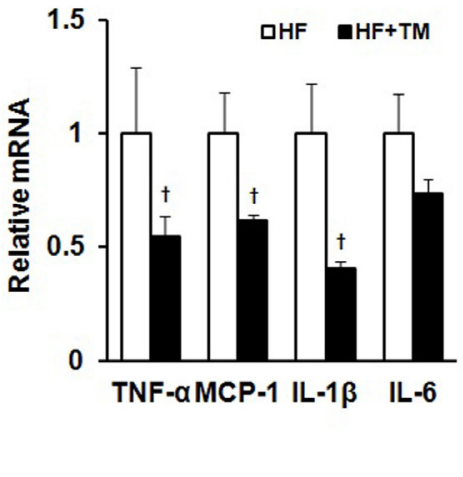

G

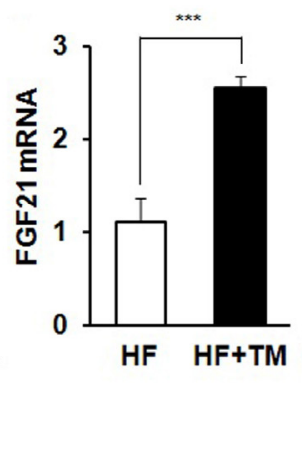

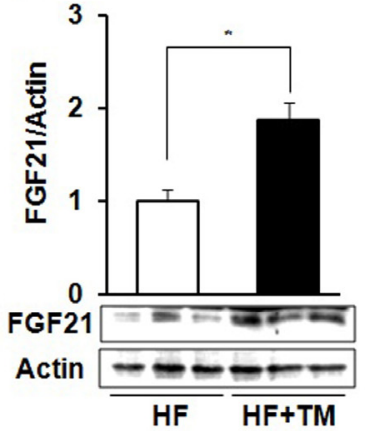

Fig. 4. TM-25659 attenuated fasting glucose and insulin sensitivity in high fat (HF) diet-induced obese mice. C57BL/6J mice were fed a HF diet for 30 weeks. They were then separated into two groups and given either vehicle or TM-25659 (50 mg/kg) orally for 14 days. (A) Body weight was measured every other day. (B) Food intake was similar between the HF and HF + TM groups. (C) Fasting blood glucose levels. (D) Area under the curve of the intraperitoneal insulin tolerance test (ITT) performed 14 days after TM-25659 treatment. (E) Representative immunoblots showing phospho AKT(Ser 473) and total AKT in the skeletal muscle of the HF and HF + TM group mice. (F) TNF- $\alpha$, IL-1 $1 \beta$, IL-6, and MCP-1 mRNA expression as quantified by real-time PCR. (G) The mRNA expression of FGF21 as quantified by real-time PCR. (H) Protein levels of FGF21 by immunoblotting (HF, HF + TM-25659). The data are given as the mean \pm standard error. ${ }^{*} P<0.05,{ }^{\star \star} P<0.01,{ }^{\star \star \star} P<$ $0.001, \dagger P<0.05, \dagger \dagger P<0.01$, vs. vehicle in the HF group mice; $n=10 /$ group.

the beneficial effects of TM-25659 must be associated with increased FGF21 in skeletal muscle.

Improved body weight following treatment with TM-25659 has been reported (Jang et al., 2012), but neither musclespecific insulin sensitivity nor the relationship between FGF21 and TM-25659 has been examined. FGF21 is a metabolic hormone produced mainly by the liver, but it is also expressed in muscle and adipose tissue where it regulates glucose and lipid homeostasis via pleiotropic actions (Woo et al., 2013). Here, we first demonstrated that TM-25659 increased FGF21 RNA and protein levels directly as dose- and time-dependent variables, and that FGF21 was secreted at significantly increased levels into mouse skeletal muscle cell medium. To test these effects in mice, TM-25659 was injected into C57BL6J mice every other day for 14 days. The FGF21 levels were dramatically augmented in biopsied muscles. Treatment of TM25659 in obese mice led to improved insulin resistance and inflammation by FGF21 induction. For long-term treatment of TM-25659 (more than 8 weeks), it stimulated weight loss (data not shown). Kim et al. (2013) demonstrated that FGF21 was induced through the translocation of activating transcription factor 4 (ATF4) from the cytoplasm to the nucleus in autophagy-deficient skeletal muscle. In addition, FGF21 was found to be induced in the liver under leucine or methionine deprivation through the GCN2-phospho-elF2 $\alpha$ and ATF4 pathways (De
Sousa-Coelho et al., 2013; Lees et al., 2014). TM-25659induced FGF21 reacts in the same way in the GCN2-phosphoelF $2 \alpha$ and ATF4 pathways.

TM-25659 improved insulin resistance in HF diet-fed mouse muscle. Mounting evidence suggests that macrophage infiltration and accumulation of chronic inflammatory cytokines such as TNF- $\alpha$, IL-1 $\beta$, IL-6, or MCP- 1 in adipose tissue is positively correlated with insulin resistance (Huh et al., 2014; Ota, 2013; Park et al., 2015; Xu et al., 2003). Because TM-25659 reduced inflammatory cytokines such as TNF- $\alpha, \mathrm{IL}-1 \beta, \mathrm{IL}-6$, and MCP-1, TM-25659 is protective against insulin resistance in mice fed an HFD. Another cause of reduced insulin resistance was FGF21 expression following TM-25659 treatment of muscle. Xu et al. (2009) reported that an FGF21 infusion given to DIO mice protected against insulin resistance by enhancing EE and lipid oxidation. Furthermore, transgenic mice overexpressing hepatic FGF21 were protected from HF diet-induced insulin resistance (Inagaki et al., 2007; Kharitonenkov et al., 2005). However, FGF21 knockdown mice had reduced adiponectin levels and aggravated chronic inflammation compared to HF diet-fed mice (Lin et al., 2013). The present study demonstrates a similar phenomenon in muscle. TM-25659 treatment prevented PAinduced insulin resistance and the induction of pro-inflammatory processes in skeletal muscle, but FGF21 siRNA eliminated the beneficial effects of TM-25659. Therefore, TM-25659 caused 
the accumulation of FGF21 in skeletal muscle and protected against insulin resistance and chronic inflammation in muscles.

In conclusion, our results suggest that TM-25659 administered to HF diet-induced obese mice prevented not only insulin resistance, but also PA-induced impaired insulin signaling. These beneficial effects were mediated via the induction of FGF21 expression.

Note: Supplementary information is available on the Molecules and Cells website (www.molcells.org).

\section{ACKNOWLEDGMENTS}

This study was supported by a grant of the Korea Healthcare technology R\&D Project, Ministry of Health and Welfare, Republic of Korea (HI12C1006/A121102).

\section{REFERENCES}

Anthony, T.G., McDaniel, B.J., Byerley, R.L., McGrath, B.C., Cavener, D.R., McNurlan, M.A., and Wek, R.C. (2004). Preservation of liver protein synthesis during dietary leucine deprivation occurs at the expense of skeletal muscle mass in mice deleted for elF2kinase GCN2. J. Biol. Chem. 279, 36553-36561.

Badman, M.K., Pissios, P., Kennedy, A.R., Koukos, G., Flier, J.S., and Maratos-Flier, E. (2007) Hepatic fibroblast growth factor 21 is regulated by PPAR alpha and is a key mediator of hepatic lipid metabolism in ketotic states. Cell Metab. 5, 426-437.

De Sousa-Coelho, A.L., Relat, J., Hondares, E., Pérez-Martí, A., Ribas, F., Villarroya, F., Marrero, P.F., and Haro, D. (2013) FGF21 mediates the lipid metabolism response to amino acid starvation. J. Lipid Res. 54, 1786-1797.

Fisher, F.M., Chui, P.C., Antonellis, P.J., Bina, H.A., Kharitonenkov, A., Flier, J.S., and Maratos-Flier, E. (2010). Obesity is a fibroblast growth factor 21 (FGF21)-resistant state. Diabetes 59, 2781-2789.

Ge, X., Wang, Y., Lam, K.S., and Xu, A. (2012). Metabolic actions of FGF21: molecular mechanisms and therapeutic implications. Acta Pharmaceutica Sinica B. 2, 350-357.

Guo, F., and Cavener, D.R. (2007). The GCN2 elF2alpha kinase regulates fatty-acid homeostasis in the liver during deprivation of an essential amino acid. Cell Metabol. 5, 1-12.

Hong, J.H., Hwang, E.S., McManus, M.T., Amsterdam, A., Tian, Y., Kalmukova, R., Mueller, E., Benjamin, T., Spiegelman, B.M., Sharp, P.A., et al. (2005) TAZ, a transcriptional modulator of mesenchymal stem cell differentiation. Science 309, 1074-1078.

Huh J.Y., Park Y.J., Ham M., and Kim J.B. (2014) Crosstalk between adipocytes and immune cells in adipose tissue inflammation and metabolic dysregulation in obesity. Mol. Cells 37, 365371

Inagaki, T., Dutchak, P., Zhao, G., Ding, X., Gautron, L., Parameswara, V., Li, Y., Goetz, R., Mohammadi, M., Esser, V., et al. (2007). Endocrine regulation of the fasting response by PPAR alpha-mediated induction of fibroblast growth factor 21. Cell Metab. 5, 415-425.

Jang, E.J., Jeong, H., Kang, J.O., Kim, N.J., Kim, M.S., Choi, S.H., Yoo, S.E., Hong, J.H., Bae, M.A., and Hwang, E.S. (2012). TM25659 enhances osteogenic differentiation and suppresses adi- pogenic differentiation by modulating the transcriptional coactivator TAZ. Br. J. Pharmacol. 165, 1584-1594.

Jung, J.G., Choi, S.E., Hwang, Y.J., Lee, S.A., Kim, E.K., Lee, M.S. Han, S.J., Kim, H.J., Kim, D.J., Kang, Y., et al. (2011). Supplementation of pyruvate prevents palmitate-induced impairment of glucose uptake in C2 myotubes. Mol. Cell Endocrinol. 345, 7987.

Kanai, F., Marignani, P.A., Sarbassova, D., Yagi, R., Hall, R.A., Donowitz, M., Hisaminato, A., Fujiwara, T., Ito, Y., Cantley, L.C. et al. (2000). TAZ: a novel transcriptional co-activator regulated by interactions with 14-3-3 and PDZ domain proteins. EMBO J. 19, 6778-6791.

Kharitonenkov, A., Shiyanova, T.L., Koester, A., Ford, A.M., Micanovic, R., Galbreath, E.J., Sandusky, G.E., Hammond, L.J., Moyers, J.S., Owens, R.A., et al. (2005). FGF-21 as a novel metabolic regulator. J. Clin. Invest. 115, 1627-1635.

Kim, K.H., Jeong, Y.T., Oh, H., Kim, S.H., Cho, J.M., Kim, Y.N., Kim S.S., Kim, D.H., Hur, K.Y., Kim, H.K., et al. (2013). Autophagy deficiency leads to protection from obesity and insulin resistance by inducing Fgf21 as a mitokine. Nat. Med. 19, 83-92.

Laeger, T., Henagan, T.M., Albarado, D.C., Redman, L.M., Bray, G.A., Noland, R.C., Münzberg, H., Hutson, S.M., Gettys, T.W., Schwartz, M.W., et al. (2014). FGF21 is an endocrine signal of protein restriction. J. Clin. Invest. 124, 3913-3922.

Lees, E.K., Król, E., Grant, L., Shearer, K., Wyse, C., Moncur, E., Bykowska, A.S., Mody, N., Gettys, T.W., and Delibegovic, M (2014). Methionine restriction restores a younger metabolic phenotype in adult mice with alterations in fibroblast growth factor 21. Aging Cell. 13, 817-827.

Lin, Z., Tian, H., Lam, K.S., Lin, S., Hoo, R.C., Konishi, M., Itoh, N., Wang, Y., Bornstein, S.R., Xu, A., et al. (2013). Adiponectin mediates the metabolic effects of FGF21 on glucose homeostasis and insulin sensitivity in mice. Cell Metab. 17, 779-789.

Ota, T. (2013). Chemokine systems link obesity to insulin resistance. Diabetes Metab. J. 37, 165-172.

Park, S., Sadanala K.C., and Kim E.K. (2015). A metabolomic approach to understanding the metabolic link between obesity and diabetes. Mol. Cells 38, 587-596

Wang, W.F., Li, S.M., Ren, G.P., Zheng, W., Lu, Y.J., Yu, Y.H., Xu, W.J., Li, T.H., Zhou, L.H., Liu, Y., et al. (2015) Recombinant murine fibroblast growth factor 21 ameliorates obesity-related inflammation in monosodium glutamate-induced obesity rats. Endocrine 49, 119-129.

Wilson, G.J., Bunpo, P., Cundiff, J.K., Wek, R.C., and Anthony, T.G. (2013). The eukaryotic initiation factor 2 kinase GCN2 protects against hepatotoxicity during asparaginase treatment. Am J. Physiol. Endocrinol. Metab. 305, 1124-1133.

Woo, Y.C., Xu, A., Wang, Y., and Lam, K.S. (2013). Fibroblast growth factor 21 as an emerging metabolic regulator: clinical perspectives. Clin. Endocrinol. 78, 489-496.

Xu, H., Barnes, G.T., Yang, Q., Tan, G., Yang, D., Chou, C.J., Sole, J., Nichols, A., Ross, J.S, Tartaglia, L.A., et al. (2003). Chronic inflammation in fat plays a crucial role in the development of obesity-related insulin resistance. J. Clin. Invest. 112, 1821-1830.

Xu, J., Lloyd, D.J., Hale, C., Stanislaus, S., Chen, M., Sivits, G., Vonderfecht, S., Hecht, R., Li, Y.S., Lindberg, R.A., et al. (2009) Fibroblast growth factor 21 reverses hepatic steatosis, increases energy expenditure, and improves insulin sensitivity in dietinduced obese mice. Diabetes 58, 250-259. 